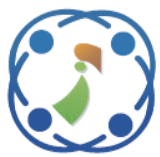

\title{
Convergence Factor and Position Updating Improved Grey Wolf Optimization for Multi-constraint and Multipath QoS Aware Routing in Mobile Adhoc Networks
}

\author{
Sulaiman Abdo Mahyoub Ghaleb ${ }^{1 *}$ \\ Vasanthi Varadharajan ${ }^{2}$ \\ ${ }^{I}$ Computer Science Department, Rathinam College of Arts and Science, Bharathiar University, Coimbatore, India \\ ${ }^{2}$ Department of Computer Technology, Sri Krishna Adithya College of Arts and Science, \\ Bharathiar University, Coimbatore, India \\ * Corresponding author's Email: sulaimanabdo2017@ gmail.com
}

\begin{abstract}
Optimal shortest path routing is one of the most common problems in Mobile adhoc networks (MANETs). Energy and delay are the two vital factors that determine the optimal shortest paths for multipath communication. Although many algorithms have tried to solve this shortest path problems, the cost and link disconnection are mostly neglected in real-time applications. This paper provokes the thought of including the multiple Quality-of-Service (QoS) parameters such as energy, delay, link quality and network lifetime as the objective parameters in selecting the optimal shortest paths. Modelling the objective cost function as an optimization problem based on these parameters, the Multi-objective Improved Grey Wolf Optimization (IGWO) algorithm based Dynamic Source Routing (DSR) protocol is developed to resolve it. IGWO is designed by modifying the location updating equations and the convergence factor to resolve the GWO local optimum problem and improve its convergence speed. First, the possible paths in the network are discovered and the fitness is evaluated for each routing path. Then the paths are sorted using the IGWO algorithm and then the optimal paths are determined with increased energy efficiency and reduced delay. Experimental results indicate the efficiency of the proposed IGWO-DSR protocol through $8.4 \%$ less delay, 3\% high throughput, $11 \%$ less energy consumption, 14\% increased lifetime, and reduced hop count by 1 and $15 \mathrm{~dB}$ increased PSNR than the original GWO algorithm. The proposed IGWO-DSR also outperforms the other existing routing algorithms.
\end{abstract}

Keywords: Mobile adhoc networks, Dynamic source routing, Shortest path, Optimal routing, Improved grey wolf optimization, Convergence rate, Local optimum problem.

\section{Introduction}

Mobile adhoc networks (MANETs) is extensively utilized in various fields for applications of healthcare, military communication, video conferences, disaster management and many other scenarios where the audio/video communication is prioritized [1]. With the advancements in Internetof-Things (IoT), the suggestion to use MANETs in smart devices are encouraged to enhance the communication benefits. However, the increasing demand of the audio/video data transmission process, the QoS must be satisfied in such a way that the uninterrupted service is ensured [2]. The challenges in assuring QoS related to the lack of central management, internal failures and external interferences [3]. The internal failures include the link breaks, battery failure, high traffic rate, process failures and packet retransmissions. The primary challenge in routing is the mobility of nodes. Generally, the MANET nodes are autonomous and are capable of dynamically identifying their own topology for forming the paths to route the packets in multi-hop manner [4]. As the MANET nodes are not stationary, the link breaks are common and routing paths are dynamically changed resulting in the increased packet drops. This link break and frequent path changes reduced the packet delivery 
ratio and degraded the reliability. Enhancing the longevity of the routing paths can be efficient in reducing the packet drops and improving reliability [5]. The other most vital challenge is the energy and delay constraints in battery operated MANET nodes. The battery failure will affect the entire network and also degrade the network lifetime. When ensuring the quality communication between the nodes, the resources such as bandwidth must be managed effectively to ensure high productivity. However, these issues lead to increased delay, high packet loss rate and reduced throughput [6]. Thus it is necessary to develop new efficient routing protocols to support the energy constraint and dynamically changing MANETs to improve the QoS.

Traditional routing algorithms namely Ad hoc On-demand Distance Vector (AODV) [7] and Dynamic Source Routing (DSR) [8] are efficient single path routing models for identifying the shortest routing paths. Nonetheless, these shortest paths are not often the optimal paths and lack stability due to excessive load and congestion, causing degraded QoS performance. Hence the routing algorithms must not only depend on the distance but also on other QoS metrics such as energy, delay, lifetime and link quality. Considering such multiple parameters can lead to paretooptimality problem and can be solved using optimization based routing protocols. On the other hand, the link failures or node failures can cause reliability issues which are common in single path routing models. Multipath routing can be the efficient solution as it provides alternate paths with consideration of the dynamic network changes. In addition to reliability, the multipath routing also reduces delay for path switching through the backup routes and improves load balancing, fault-tolerance and high throughput [9]. Therefore, the development of multipath routing based multi-objectives is formulated as the main objective in the design of new efficient MANET routing models.

Many optimization based routing protocols have been developed in recent years for achieving this objectives. Although efficient at achieving the desired outcome, some limitations such as slow convergence due to local optimum problem reduced the optimal performance. Previously, Multiobjective Grey Wolf Optimization based Dynamic Source Routing (MGWO-DSR) protocol [10] was developed to provide efficient multipath routing based on multiple QoS objectives. While significant results were obtained, the extensive analysis has suggested that the GWO may also suffer from the local optimum problem when the network size and mobility rate increases. To tackle this problem, this paper proposes multi-objective IGWO-DSR routing model in which the GWO is modified by introducing new position updating equation and convergence factor to influence the balance between the exploration and exploitation. The position updating strategy is adaptive to the individual search agent fitness and hence the comparisons will be faster and accurate. Similarly, the convergence factor in GWO is bi-linear which is modified into non-linear equation in IGWO to characterize the wolves' movement in all directions. These two improvements in IGWO increase the convergence rate and improve the global search process to obtain best solutions that are more ideal than that obtained using the GWO. The main advantage in using IGWO-DSR over the GWO is the efficiency in obtaining better convergence characteristics that reduces the delay and improves the optimal route selection process. Experimental results are performed to evaluate the superior performance of the multi-objective IGWO-DSR over its counterpart models. The rest of the article is organized as: Section 2 discusses some recent existing routing models. Section 3 explains the proposed IGWODSR protocol and section 4 presents the evaluation results. Section 5 makes a conclusion of this research study.

\section{Related works}

Optimization based routing algorithms have been gained immense interest in the research field for different network models. MANETs consisting of mobile nodes have predominantly relied on optimal route selection based on QoS metrics. Rajadurai et al. [11] developed multicast ad hoc ondemand routing using genetic algorithm based on packet deliver ratio, latency and energy for multipath selection. Though it is efficient, the reliability issues and slow data rate due to global congestion reduces the overall effectiveness. Tareq et al. [12] employed Artificial bee colony (ABC) based DSR model with energy, node speed and packet size as objective parameters. However, the energy consumption is only slightly minimized due to the support for only single path routing. Wei et al. [13] developed multi-objective multicast routing using differential evolution and used network lifetime, cost, delay, jitter and bandwidth as objectives for route selection. Although it has efficient performance with higher stability, this routing model has reduced the network throughput. Zhang et al. [14] designed a DSR model based on genetic algorithm-bacterial foraging optimization (GA-BFO) with the network lifetime, hop count and 
energy as objectives. Still the GA-BFO algorithm has limitations in convergence speed. Krishna et al. [15] developed hybrid PSO-GA optimisation algorithm for energy efficient routing based on energy, delay and packet delivery ratio.

Prasath and Sreemathy [16] proposed Firefly algorithm (FA) DSR model depending on link quality, node mobility and delay. Although this approach improved throughput and reduced the delay and retransmissions, the number of hops were increased greatly resulting in energy wastage. Robinson et al. [17] proposed Particle swarm optimization-based multipath routing using the bandwidth and link availability prediction to minimize the resource utilization and increase the QoS. This approach employs fuzzy logic to select the optimal forwarding nodes with minimum polynomial time. The limitation of adapting to larger network is still a problem for this approach. Reddy [18] suggested a multi-path QoS-aware routing algorithm using the fractional cuckoo search algorithm based on energy, link life time, distance and delay parameters. However, the performance of this approach reduced due to the poor convergence speed when link failures occur. Sekar and Mangalam [19] created a third generation selfgeneration and co-evolution based memetic optimization to develop energy and load balancing aware routing model. This model reduces the overhead and increases the packet delivery ratio and load balancing metric but has shortcomings of the mutation operators.

Malar et al. [20] introduced multi constraint based energy efficient routing Ant Colony Optimization (ACO) that reduces the energy consumption and adapts itself to adverse environments. Junnarkar et al. [21] also developed ACO based QoS and mobility aware multipath opportunistic routing to ensure stability and longer network lifetime. Still the convergence rate of ACO for larger networks is not efficient and hence improvements are required. Mukhedkar and Kolekar [22] proposed encrypted trust-based dolphin glowworm optimization (E-TDGO) for designing secure routing to minimize delay, packet drop and improve the attack detection and throughput. However, the hybrid optimization and encryption processes increase the computation complexity of this model. From the literature studies, it is inferred that the major problems in optimization based routing models is the local optimum problem and slow convergence rate. Apart from the common QoS performance issues, these optimization problems take the center-stage. Hence this paper has strived to develop an improved GWO algorithm for energy efficient QoS aware routing model based on multiple QoS parameters.

\section{Methodology}

In the proposed multi-objective IGWO-DSR model for QoS aware routing, the traditional DSR routing model is optimally integrated by the improved GWO algorithm to enhance the routing performance based on QoS metrics. Table 1 presents the list of notations used in this paper and their definitions.

Table 1. Notations and definitions

\begin{tabular}{|c|c|}
\hline Notation & Definition \\
\hline$F(x)$ & Multi-objective fitness function \\
\hline$w_{1}, w_{2}, w_{3}, w_{4}$ & Weight values \\
\hline$i$ & Data packet \\
\hline$e_{t}$ & Transmission energy of packet $i$ \\
\hline$e_{r}$ & Reception energy of packet $i$ \\
\hline$P$ & $\begin{array}{l}\text { constant continuous power } \\
\text { consumption }\end{array}$ \\
\hline$t$ & time \\
\hline$T_{r i}$ & Receive time of $i$-th packet \\
\hline$T_{s i}$ & Sending time of $i$-th packet \\
\hline$n$ & Total number of data packets \\
\hline$d$ & $\begin{array}{l}\text { Distance between source and } \\
\text { destination nodes }\end{array}$ \\
\hline$\varepsilon_{0}$ & $\begin{array}{l}\text { total non-rechargeable initial } \\
\text { energy }\end{array}$ \\
\hline$\lambda$ & average broadcasting rate \\
\hline $\mathbb{E}\left[E_{w}\right]$ & expected unused energy \\
\hline $\mathbb{E}\left[E_{r}\right]$ & expected reporting energy \\
\hline$L_{q}$ & Link quality \\
\hline$A G_{r}$ and $A G_{t}$ & $\begin{array}{l}\text { antenna gain of receiver and } \\
\text { transmitter }\end{array}$ \\
\hline$P_{r}$ and $P_{t}$ & receiver and transmitter power \\
\hline$\alpha, \beta, \delta, \omega$ & grey wolf search agents \\
\hline$\vec{O}$ & enclosing model \\
\hline$\vec{D}$ and $\vec{B}$ & coefficient vectors \\
\hline$\vec{Y}_{k}$ & position vectors of the prey \\
\hline$\vec{Y}(m)$ & position vectors of the grey wolf \\
\hline$m$ & Number of iterations \\
\hline$m_{\max }$ & Maximum iterations \\
\hline$\vec{c}$ & Convergence factor \\
\hline$\vec{r}_{1}, \vec{r}_{2}$ & Random numbers \\
\hline$\vec{Y}_{1}, \vec{Y}_{2}$ and $\vec{Y}_{3}$ & best position vectors \\
\hline$\vec{Y}_{\alpha}, \vec{Y}_{\beta}$ and $\vec{Y}_{\delta}$ & 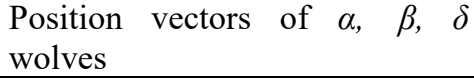 \\
\hline$\vec{O}_{\alpha}, \vec{O}_{\beta}$ and $\vec{O}_{\delta}$ & $\begin{array}{l}\text { enclosing model for distance } \\
\text { between the grey wolves and the } \\
\text { position vector }\end{array}$ \\
\hline$\theta$ & Control parameter \\
\hline$f_{\alpha}, f_{\beta}, f_{\delta}$ & $\begin{array}{l}\text { fitness of alpha, beta and delta } \\
\text { wolves }\end{array}$ \\
\hline$F$ & $\begin{array}{l}\text { cumulative sum of } f_{\alpha}, f_{\beta}, f_{\delta} \\
\text { values }\end{array}$ \\
\hline
\end{tabular}

DOI: $10.22266 /$ ijies2020.0831.40 


\subsection{Dynamic source routing}

DSR is the reactive source routing approach that identifies the paths from the source node only when the data transmission is needed. DSR performs the unicast routing where the source node can transmit data only to one destination node at a time. In DSR unicast routing, the cache memory of the nodes store the routing details of the latest paths used by that specific node. Based on these routing table data, the routes are discovered and maintained at each phase. The source nodes check the routing cache table before transmitting data packets to the destination nodes. The packets are transmitted only when the reliable routes are available between the two nodes while the transmission is prohibited temporarily when no routes are available, to accommodate the route discovery process.

The route discovery is performed by the broadcasting of route request (RREQ) packets. The nodes receiving the RREQ analyses its cache table and when there is no prior route information about the sender node, the receiving node adds its own address to the packet and broadcasts to the other neighbouring nodes. In case, there is prior route information about the sender node and is the neighbour to the destination node or having prior routing history with the destination, then the route reply (RREP) packet is created. The RREP packet generated at the destination consists of all the node addresses that appended their address to the RREQ packet. Using this traversed history, the routes can be formed through all the intermediate nodes and the one with the shortest path is selected for transmission.

The nodes maintain the route information through the RREP packets and the ROUTE_ERROR packets. ROUTE_ERROR packets are generated by the nodes when the data link layer finds breaks or disconnection in the paths that causes the link failure. Once the error packet is generated, the original data packets are transmitted again from the point of link failure to the source node in the reverse direction. At this case, all the intermediate nodes will eliminate the failed link from the routing table and the source nodes begin fresh route discovery process. The control overheads in route discovery are highly reduced in DSR. However, the DSR cannot handle larger size packet headers as it is suitable for source based routing i.e. the efficiency of routing decreases when the RREQ packets are transmitted far from the source node when the search for routing to destination node intensifies. This limitation can be rectified by optimizing the DSR model using IGWO. In IGWO-DSR, the paths are assigned as the wolves and the optimal path is selected based on the fitness values.

\subsection{Fitness function formation}

The parameters namely energy, delay, lifetime, and link quality are considered to form the fitness function for determining the best paths in the proposed IGWO-DSR. At time $t$, the multi-objective function is modelled as

$$
\begin{aligned}
& F(x)=w_{1} \times \text { Energy }+w_{2} \times \text { delay }+ \\
& w_{3} \times \text { Lifetime }+w_{4} \times \text { Link quality }
\end{aligned}
$$

Here $w_{1}, w_{2}, w_{3}$ and $w_{4}$ are the weight values assigned to the four parameters which are computed for each available route. The values for $w_{1}, w_{2}$, $w_{3}$ and $w_{4}$ are chosen such that $w_{1}, w_{2}, w_{3}, w_{4}>0$ and $w_{1}+w_{2}+w_{3}+w_{4}=1$. The four objective parameters are computed based on the following Eqs. (2)-(6).

$$
\operatorname{Energy}(e)=(2 \times i-1)\left(e_{t}+e_{r}\right) d
$$

Here $i$ denotes the data packet, $e_{t}$ represents the energy for transmission of packet $i, e_{r}$ is the energy for receiving the packet $i$ and $d$ is the distance between transmission node and destination node.

$$
\text { Average delay }\left(T_{a d}\right)=\frac{\sum_{i=1}^{n}\left(T_{r i}-T_{s i}\right)}{n}
$$

Here $T_{r i}$ and $T_{s i}$ denote the receive time and sending time, respectively, of $\mathrm{i}$-th data packet, and $n$ represent the total number of packets.

$$
\text { Lifetime } \mathbb{E}[L]=\frac{\varepsilon_{0}-\mathbb{E}\left[E_{w}\right]}{P+\lambda \mathbb{E}\left[E_{r}\right]}
$$

Here the constant continuous power consumption of the network is represented as $\mathrm{P}, \varepsilon_{0}$ is the total nonrechargeable initial energy, $\lambda$ is the average broadcasting rate, $\mathbb{E}\left[E_{w}\right]$ and $\mathbb{E}\left[E_{r}\right]$ are the expected wasted energy and the expected broadcasting energy, respectively.

$$
\begin{aligned}
& \text { Link Quality }\left(L_{q}\right) \propto P_{r} \\
& P_{r}=P_{t} \times A G_{r} \times A G_{t} \times \frac{\lambda^{2}}{(4 \pi d)^{2}}
\end{aligned}
$$

Here $P_{r}$ and $P_{t}$ are the receiver and transmitter power respectively, $A G_{r}$ and $A G_{t}$ are the antenna gain of receiver and transmitter respectively. 


\subsection{Grey wolf optimization algorithm}

Grey wolf optimization (GWO) [23] is a metaheuristic algorithm which mimics the organized hunting structure and social hierarchy of the grey wolves. In GWO, grey wolves are characterized into alpha $(\alpha)$, beta $(\beta)$, delta $(\delta)$ and omega $(\omega)$ according to their social hierarchy. The alpha wolves are the leader packs and the beta wolves are the second level category of co-leaders. The delta wolves are the third class acting as elders while the omega wolves are the reserve wolves' back-up and have to wait for their act. Based on this hierarchy, the optimization procedure is formulated depending upon the hunting behaviour of each pack of wolves. The hunting behaviour of each class of wolves can be represented mathematically as

$$
\begin{aligned}
& \vec{O}=\left|\vec{B} \cdot \vec{Y}_{k}(m)-\vec{Y}(m)\right| \\
& \vec{Y}(m+1)=\vec{Y}_{k}(m)+\vec{D} \cdot \vec{O}
\end{aligned}
$$

Here $\vec{Y}_{k}$ and $\vec{Y}(m)$ are the position vectors of the prey and the grey wolf, respectively, at $\mathrm{m}$-th iteration and $\vec{O}$ is the enclosing model. $\vec{D}$ and $\vec{B}$ represent the coefficient vectors which are computed as

$$
\begin{aligned}
& \vec{D}=2 \vec{c} \cdot \vec{r}_{1}-\vec{c} \\
& \vec{B}=2 \cdot \vec{r}_{2}
\end{aligned}
$$

Here $\vec{c}$ is convergence factor and it is linearly decreased from 2 to 0 at each iteration to improve the convergence rate and random numbers $\vec{r}_{1}, \vec{r}_{2} \in$ $[0,1]$. The hunting process is represented by Eqs. (11) - (16).

$$
\begin{aligned}
& \left.\vec{O}_{\alpha}=\mid \vec{B}_{1} \cdot \vec{Y}_{\alpha}(m)-\vec{Y}\right) \mid \\
& \left.\vec{O}_{\beta}=\mid \overrightarrow{B_{2}} \cdot \vec{Y}_{\beta}(m)-\vec{Y}\right) \mid \\
& \left.\vec{O}_{\delta}=\mid \vec{B}_{3} \cdot \vec{Y}_{\delta}(m)-\vec{Y}\right) \mid \\
& \vec{Y}_{1}=\vec{Y}_{\alpha}-\vec{D}_{1} \cdot\left(\vec{O}_{\alpha}\right) \\
& \vec{Y}_{2}=\vec{Y}_{\beta}-\vec{D}_{1} \cdot\left(\vec{O}_{\beta}\right) \\
& \vec{Y}_{3}=\vec{Y}_{\delta}-\vec{D}_{1} \cdot\left(\vec{O}_{\delta}\right)
\end{aligned}
$$

Here $\vec{Y}_{1}, \vec{Y}_{2}$ and $\vec{Y}_{3}$ are the best position vectors of the $\vec{Y}_{\alpha}, \vec{Y}_{\beta}$ and $\vec{Y}_{\delta}$ grey wolves respectively. $\vec{O}_{\alpha}, \vec{O}_{\beta}$ and $\vec{O}_{\delta}$ represent the enclosing model for distance between the agents alpha $(\alpha)$, beta $(\beta)$, and delta $(\delta)$, respectively, to the position $\vec{Y}$. The position update is given by

$$
Y(m+1)=\frac{\vec{Y}_{1}+\vec{Y}_{2}+\vec{Y}_{3}}{3}
$$

Based on this hunting and exploitation behaviour, the multi-objective GWO [24] can be formulated by adding multiple constraints to the fitness function. Algorithm 1 illustrates the optimization process using GWO.

\section{Algorithm 1. GWO algorithm}

Begin

Initialize grey wolf population $X i$

Initialize $\vec{c}, \vec{D}$ and $\vec{B}$

Estimate the fitness for each wolf agent

Rank the wolf agents based on fitness as $\vec{Y}_{\alpha}, \vec{Y}_{\beta}$ and $\vec{Y}_{\delta}$

While ( $m<$ maximum iterations)

For each grey wolf End for

Update the position by Eq. (17)

Update $\vec{c}, \vec{D}$ and $\vec{B}$

Compute fitness for all grey wolves after position update

Re-rank $\vec{Y}_{\alpha}, \vec{Y}_{\beta}$ and $\vec{Y}_{\delta}$ wolf order

End while

Increment $m$ by 1

Return $\vec{Y}_{\alpha}$

End

\subsection{Improved GWO algorithm}

GWO has better convergence rate as the global search process is effectively performed. However, this holds true only for small scale routing problems. When the network size increases or the mobility rate of the node varies, the global search process of GWO is truncated. This might result in reduced convergence rate and returning the local optimum solution as the global optimum solution. This problem can indirectly affect the routing performance in MANETs. Hence effective strategies must be attained to resolve these limitations of GWO.

The limitation of GWO can be eliminated by improving the global search process. Through extensive analysis, it is found that the convergence factor $\vec{c}$ plays an important role in stabilizing the 
convergence rate of GWO. This convergence factor is linearly decreased and is limited towards only one direction of wolf movement. Modifying this linear factor into non-linear vector form can enhance the search process in all directions. Another finding is that the position-update equation of GWO contains the position vectors of the respective wolf classes. Modifying this equation by introducing the individual fitness as well as cumulative fitness of the grey wolves can have greater impact. These two changes are made in the original GWO to form the IGWO algorithm. First modification of $\vec{c}$ is given as

$$
\vec{c}=\theta e^{-2\left(1-\frac{m}{m_{\max }}\right)}
$$

Here $\theta$ is the control parameter to convert linear $\vec{c}$ into non-linear vector and $m_{\max }$ is the maximum number of iterations. Applying this new $\vec{c}$ to GWO modifies the Eq. (9) as given in Eq. (19).

$$
\vec{D}=\left[2 \cdot \vec{r}_{1}-1\right] \times \theta e^{-2\left(1-\frac{m}{m_{\max }}\right)}
$$

Similarly, applying the second change of modifying position-update equation, Eq. (17) of GWO becomes

$$
Y(m+1)=\frac{1}{3}\left[\vec{Y}_{1} \cdot \frac{f_{\alpha}}{F}+\vec{Y}_{2} \cdot \frac{f_{\beta}}{F}+\vec{Y}_{3} \cdot \frac{f_{\delta}}{F}\right]
$$

Where $f_{\alpha}, f_{\beta}, f_{\delta}$ are the fitness of alpha, beta and delta wolves calculated from $F(x)$ (Eq.1) and $F$ is the cumulative sum of $f_{\alpha}, f_{\beta}, f_{\delta}$ values. Employing the Eq. (19) and (20) in the original GWO eradicates the local optimum problem and improves the convergence rate.

\subsection{Multi-objective IGWO-DSR model}

The proposed routing algorithm of multiobjective IGWO-DSR considers the multiple parameters used in the fitness function. For achieving the multi-objective optimization, the IGWO integrates two components namely an archive and a leader selection strategy. The first component is responsible for sorting and storing the non-dominated pareto-optimal solutions as modelled in Eq. (1). The second component helps in selecting the alpha, beta and delta paths as the leaders apart from the omega paths in MANET archive of multiple paths. The best solutions are the alpha, beta and delta while the worst solution set omega is also sorted for future iterations. The leader selection policy decides the alpha, beta and delta paths centred on the selection principles and it is highly feasible to select dominant brand new solutions.
The IGWO-DSR initializes the route discovery as in traditional DSR and once the routes are known, the selection process begins by the IGWO. The optimal selection depends on the fitness value of each path assigned as individual grey wolf search agents. The paths thus selected will be highly efficient with less energy consumption, lower delay, higher lifetime and high link quality. The time complexity of the IGWO-DSR is computed as $O\left(M N^{2}\right)$ where $N$ is the number of paths (individuals) in the network and $M$ is the number of objectives. The time complexity is equal to MGWODSR algorithm and hence the benefits of IGWO are evident. Algorithm 2 summarizes the IGWO-DSR routing model.

\section{Algorithm 2. IGWO-DSR model \\ Begin \\ Initialize $\mathrm{N}$ nodes \\ Collect the nodes' initial positions \\ Randomly set the speed of each node \\ Regulate the source and destination node \\ Initiate DSR Route discovery process \\ Return non-dominant solution of paths \\ Initiate IGWO route optimization}

Generate the grey wolf population $X i(i=$ $1,2, \ldots, n)$

Determine $\vec{c}, \vec{D}$ and $\vec{B}$

Compute the objective parameters for every search agent using Eq. (2) - (6)

Assess fitness value for each path using Eq. (1)

Compute the non-dominated solutions and initialize the archive

$\vec{Y}_{\alpha}=$ Select Leader (archive)

Exclude alpha from the archive temporarily to avoid choosing the same leader

$\vec{Y}_{\beta}=$ Select Co-Leader (archive)

Exclude beta from the archive temporarily to avoid choosing the same leader

$\vec{Y}_{\delta}=$ Select Elder (archive)

Add back alpha and beta to the archive

$m=1$;

While ( $m<$ Max number of iterations)

For each grey wolf

Update the position by Eq. (20)

End for

Update $\vec{c}, \vec{D}$ and $\vec{B}$

Compute fitness after position update

Discover the several shortest routing paths

Update the archive with respect to the obtained new non-dominated solutions

Add the new solution to the archive

Calculate energy probability value 
Store the best energy routes

Re-rank $\vec{Y}_{\alpha}, \vec{Y}_{\beta}$ and $\vec{Y}_{\delta}$ wolf order

Increment $m$ by 1

End while

Return $\vec{Y}_{\alpha}$ (multiple paths)

Perform balanced data transmission from source to destination

Resort $\alpha, \beta, \delta$ paths along with omega paths

End

\section{Experimental results}

The performance of the suggested IGWO-DSR method is evaluated and compared with existing methods such as DSR, FA-DSR [16], ABC-DSR [12] and MGWO-DSR [10] in terms of end to end delay, throughput, energy consumption, hop count, PSNR and network lifetime. The experiments are conducted using NS-2 simulator. 100 nodes are distributed in an area of $1000 \times 1000 \mathrm{~m}$ with each node having initial energy of 100 Joules. The simulation settings are given in Table 2 .

Fig. 1 illustrates the comparison of (a) end to end delay, (b) throughput, (c) energy consumption, (d) network lifetime, (e) PSNR and (f) hop count results between the proposed IGWO-DSR against the existing DSR, FA-DSR, ABC-DSR and MGWO-DSR routing models. Fig. 1 (a) shows the comparison of end to end delay performance for proposed IGWO-DSR against the existing routing models. Evaluated with increasing number of nodes from 20 to 100, the proposed IGWO-DSR algorithm obtains less end to end delay than the existing DSR,

Table 2. Simulation Parameters

\begin{tabular}{|l|l|}
\hline Simulator & NS-2.34 \\
\hline No. of Nodes & 100 \\
\hline Area Size & 1000 X $1000 \mathrm{~m}$ \\
\hline Channel type & Wireless Channel \\
\hline Propagation model & Two Ray Ground \\
\hline Link Layer & LL \\
\hline Antenna model & Omni Antenna \\
\hline Traffic type & CBR \\
\hline Mobility model & Random Way point \\
\hline MAC & 802.11 \\
\hline Initial energy & 100 Joules \\
\hline Radio Range & $250 \mathrm{~m}$ \\
\hline Simulation Time & 300 seconds \\
\hline Number of packets & 1000 \\
\hline Packet rate & 8 packets/sec \\
\hline Data payload & 512 bytes/packet \\
\hline
\end{tabular}

FA-DSR, ABC-DSR and MGWO-DSR algorithms. For 100 nodes, the end-to-end delay of IGWO-DSR is $1.09 \mathrm{~ms}$ which is $8.4 \%, 19 \%, 34 \%$ and $43 \%$ reduced than the MGWO-DSR, ABC-DSR, FADSR and traditional DSR models, respectively. The reduced delay is due to the increased efficiency in shortest path selection optimally with less computation time.

Fig. 1 (b) shows that with increasing number of nodes from 20 to 100 , the proposed IGWO-DSR algorithm obtains high throughput than the existing DSR, FA-DSR and ABC-DSR algorithms. For 100 nodes, the IGWO-DSR has higher throughput of $175 \mathrm{kbps}$ which is $3 \%, 10.7 \%$ and $43 \%$ greater than the MGWO-DSR, ABC-DSR and FA-DSR models, respectively. Fig. 1 (c) shows that with increasing number of nodes from 20 to 100, the proposed IGWO-DSR algorithm obtains less energy consumption than the existing DSR, FA-DSR and ABC-DSR algorithms. For 100 nodes, the IGWODSR has consumed energy of $18.6(\mathrm{~J} / \mathrm{s})$ which is $11 \%, 26 \%, 28.7 \%$ and $34.7 \%$ less than the MGWODSR, ABC-DSR, FA-DSR and traditional DSR models, respectively. Energy reduction is achieved through the optimal energy aware route selection.

Fig. 1 (d) provides the network lifetime comparison for the IGWO-DSR against the existing routing models. Evaluated with increasing network load, the proposed IGWO-DSR algorithm obtains high network lifetime than the existing DSR, FADSR and ABC-DSR algorithms. For $1000 \mathrm{kbps}$ load, the IGWO-DSR has lifetime of 788 seconds which is $14 \%, 39 \%$ and $87 \%$ higher lifetime MGWO-DSR, ABC-DSR and FA-DSR models, respectively. As a result of reduced energy consumption, the battery reserves have been increased and the network has survived more time than the other models.

Fig. 1 (e) provides the hop count comparison of proposed IGWO-DSR against the existing routing models. Evaluated with increasing number of nodes, the proposed IGWO-DSR algorithm takes fewer hops than the existing DSR, FA-DSR and ABCDSR algorithms. For 100 nodes, the IGWO-DSR takes 1 hop, 4 hops, 6 hops and 7 hops less than the MGWO-DSR, ABC-DSR, FA-DSR and traditional DSR models, respectively. As the high number of hops can also increase the transmission time and possibility of link breaks and retransmissions, the less number of hops is ensured through the IGWO optimal routing.

Fig. 1 (f) shows that with increasing number of data frames, the proposed IGWO-DSR algorithm obtains high PSNR than the existing DSR, FA-DSR and ABC-DSR algorithms. For 100 nodes, the IGWO-DSR has $15 \mathrm{~dB}, 25 \mathrm{~dB}, 36 \mathrm{~dB}$ and $43 \mathrm{~dB}$ 
higher PSNR than the MGWO-DSR, ABC-DSR, FA-DSR and traditional DSR models, respectively,

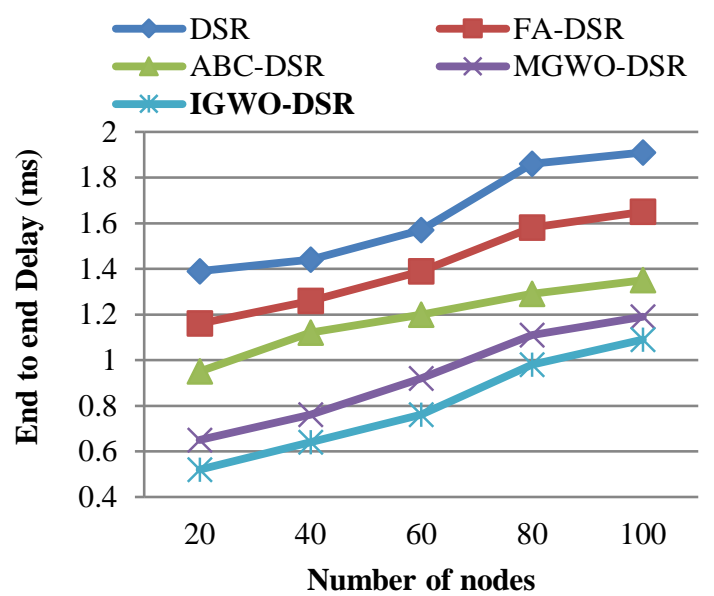

(a)

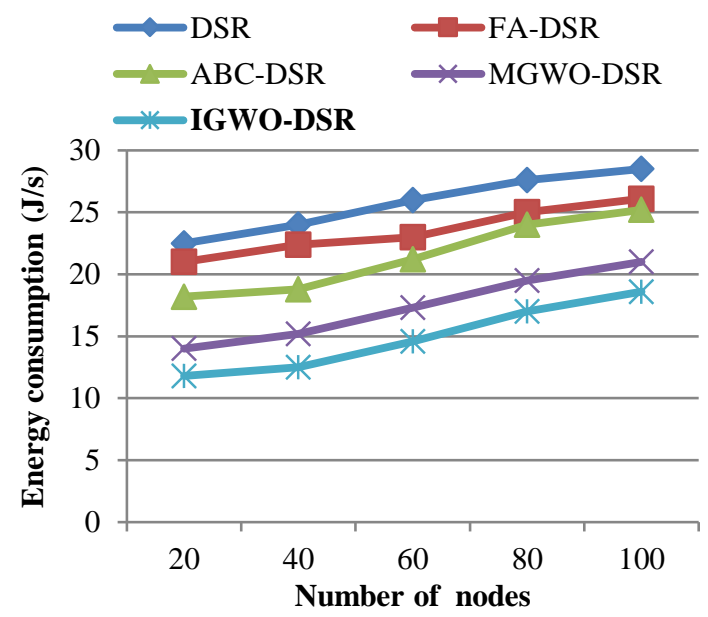

(c)

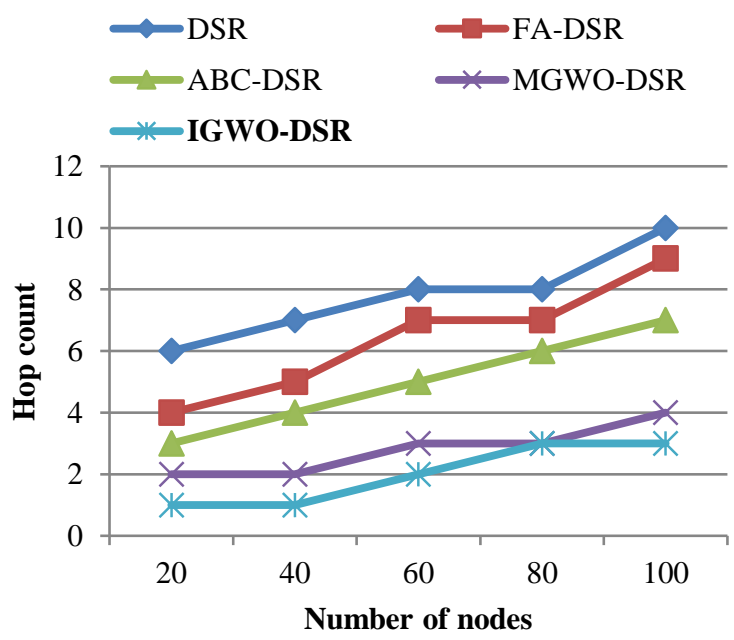

(e)

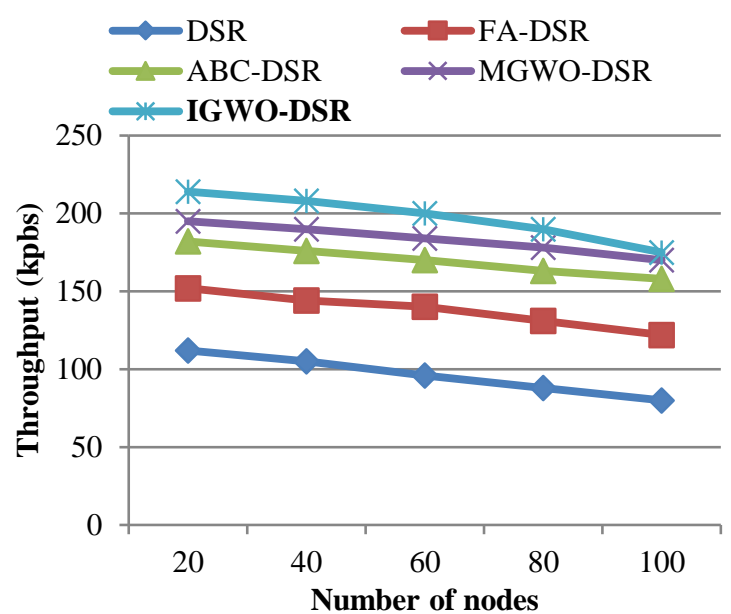

(b)

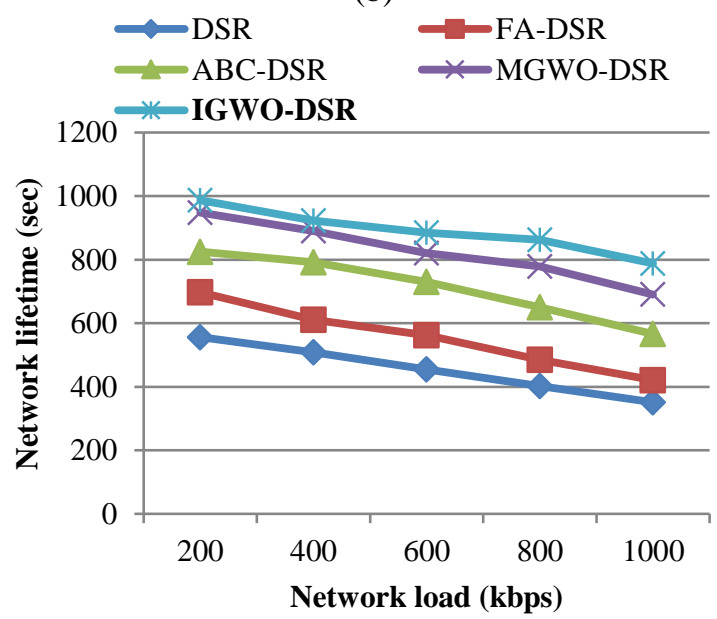

(d)

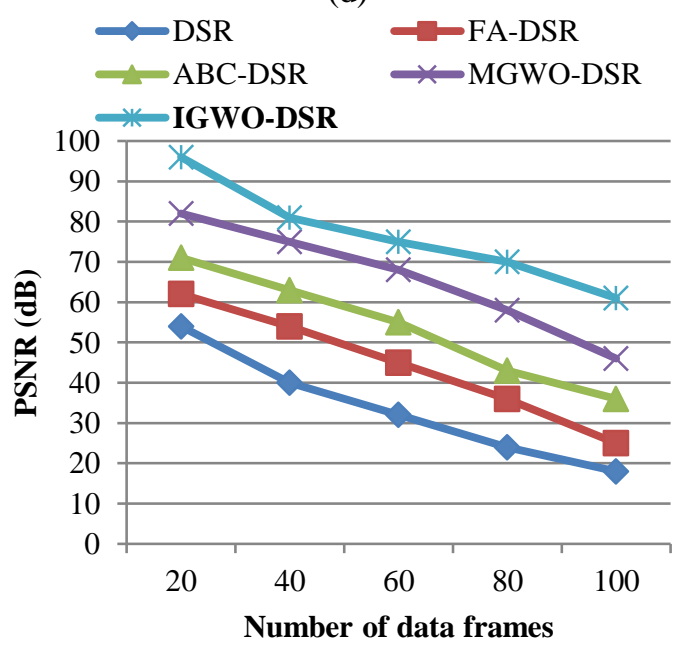

(f)

Figure. 1 Performance comparison: (a) End-to-end delay, (b) Throughput, (c) Energy consumption, (d) Network Lifetime, (e) Hop count, and (f) PSNR 
From the simulation results, it can be determined that the proposed IGWO-DSR provides better routing performance than the existing models with less delay, less energy consumption, less hop counts, higher throughput, high lifetime and higher PSNR values. Hence the multi-objective IGWO-DSR routing model can be efficient for multipath routing with multiple constraints to achieve high QoS performance in MANETs.

\section{Conclusion and future work}

Energy efficient, QoS aware multipath routing based on multiple constraints is challenging in MANETs. This paper has suggested an efficient routing model by optimizing the DSR model by Improved GWO algorithm. This IGWO-DSR model improves the convergence rate and avoids the local optimum problem of GWO algorithm to ensure proficient routing. The experiments conducted in NS-2 with 100 simulation nodes provide efficient results for IGWO-DSR with $8.4 \%$ less delay, $3 \%$ high throughput, $11 \%$ less energy consumption, $14 \%$ increased lifetime, reduced hop count by 1 and $15 \mathrm{~dB}$ increased PSNR than the previous MGWODSR algorithm. The proposed IGWO-DSR also outperforms the existing DSR, FA-DSR and ABCDSR routing algorithms and increased the QoS performance.

In future, the proposed IGWO-DSR will be extended to support large scale MANETs which consists of hundreds/thousands of active nodes. Investigations will be made to examine the possibility of including additional QoS metrics to the fitness function without increasing the overhead. Also, the utilization of hybrid optimization models for routing with minimum time complexity will be investigated.

\section{Conflicts of Interest}

The authors declare no conflict of interest.

\section{Author Contributions}

This work is a contribution of both the authors: Conceptualization, Sulaiman Abdo Mahyoub Ghaleb, and Vasanthi Varadharajan; methodology, Sulaiman Abdo Mahyoub Ghaleb; software, Sulaiman Abdo Mahyoub Ghaleb; validation, Sulaiman Abdo Mahyoub Ghaleb, and Vasanthi Varadharajan; formal analysis, Sulaiman Abdo Mahyoub Ghaleb; writing-original draft preparation, Sulaiman Abdo Mahyoub Ghaleb; writing - review and editing, Sulaiman Abdo Mahyoub Ghaleb, and Vasanthi Varadharajan.

\section{References}

[1] A. Singh, S. Batra, and P. Goyal, "Mobile Ad hoc Networks: Challenges, Applications and their Routing Protocols", International Journal on Recent Trends in Engineering and Technology, Vol. 1, No. 2, pp. 4-12, 2010.

[2] M. Kumar and R. Mishra, "An overview of MANET: history, challenges and applications", Indian Journal of Computer Science and Engineering (IJCSE), Vol. 3, No. 1, pp. 121125, 2012.

[3] S. Y. Oh, G. Marfia, and M. Gerla, "MANET QoS support without reservations", Security and Communication Networks, Vol. 4, No. 3, pp.316-328, 2011.

[4] R. R. Roy, Handbook of mobile ad hoc networks for mobility models, New York, NY, USA: Springer, Vol. 170, 2011.

[5] A. K. Gupta, H. Sadawarti, and A. K. Verma, "Performance analysis of MANET routing protocols in different mobility models", International Journal of Information Technology and Computer Science (IJITCS), Vol. 5, No. 6, pp. 73-82, 2013.

[6] S. Gopinath, N. Sureshkumar, G. Vijayalakshmi, N. A. Natraj, T. Senthil, and P. Prabu, "Energy efficient routing protocol for MANET", International Journal of Computer Science Issues (IJCSI), Vol. 9, No. 2, pp. 292297, 2012.

[7] S. Sethi, and S. K. Udgata, "Optimized and Reliable AODV for MANET", International Journal of Computer Applications, Vol. 97, No. 5, pp. 8887-8898, 2010.

[8] N. R. Patel, and S. Kumar, "Energy conscious DSR in MANET", In: Proc. of the 2nd IEEE International Conference on Parallel, Distributed and Grid Computing, pp. 784-789, 2012.

[9] S. V. Gole and S. V. Mallapur, "Multipath energy efficient routing protocol", International Journal of Research and Reviews in Computer Science, Vol. 2, No. 4, pp. 954963, 2011.

[10] S. A. M. Ghaleb and V. Vasanthi, "Energy Efficient Multipath Routing Using MultiObjective Grey Wolf Optimizer based Dynamic Source Routing Algorithm for MANET", International Journal of Advanced Science and Technology, Vol. 29, No. 3, pp. 6096-6117, 2020.

[11] S. J. G. Rajadurai, J. Veerappan, and K. Ramasamy, "Optimization of multicast ad hoc on-demand routing protocol based on genetic 
algorithm with backup paths in MANET", Wireless Personal Communications, Vol. 94, No. 4, pp. 2095-2124, 2017.

[12] M. Tareq, R. Alsaqour, M. Abdelhaq, and M. Uddin, "Mobile ad hoc network energy cost algorithm based on artificial bee colony", Wireless Communications and Mobile Computing, Vol. 2017, pp. 1-12, 2017.

[13] W. Wei, Y. Qin, and Z. Cai, "A multi-objective multicast routing optimization based on differential evolution in MANET", International Journal of Intelligent Computing and Cybernetics, Vol. 11 No. 1, pp. 121-140, 2018.

[14] D. G. Zhang, S. Liu, X. H. Liu, T. Zhang, and Y. Y. Cui, "Novel dynamic source routing protocol (DSR) based on genetic algorithmbacterial foraging optimization (GA-BFO)", International Journal of Communication Systems, Vol. 31, No. 18, p. e3824, 2018.

[15] S. R. K. M. Krishna, M. B. N. S. Ramanath, and V. K. Prasad, "Optimal reliable routing path selection in MANET through hybrid PSOGA optimisation algorithm", International Journal of Mobile Network Design and Innovation, Vol. 8, No. 4, pp. 195-206, 2018.

[16] N. Prasath and J. Sreemathy, "Optimized dynamic source routing protocol for MANETs", Cluster Computing, Vol. 22, No. 5, pp. 1239712409, 2019.

[17] Y. H. Robinson, S. Balaji, and E. G. Julie, "PSOBLAP: particle swarm optimization-based bandwidth and link availability prediction algorithm for multipath routing in mobile ad hoc networks", Wireless Personal Communications, Vol. 106, No. 4, pp. 22612289, 2019.

[18] S. Reddy, "Multi-path selection based on fractional cuckoo search algorithm for QoS aware routing in MANET", Sensor Review, Vol. 39 No. 2, pp. 218-232, 2019.

[19] P. C. Sekar and H. Mangalam, "Third generation memetic optimization technique for energy efficient routing stability and load balancing in MANET", Cluster Computing, Vol. 22, No. 5, pp. 11941-11948, 2019.

[20] A. C. J. Malar, M. Kowsigan, N. Krishnamoorthy, S. Karthick, E. Prabhu, and K. Venkatachalam, "Multi constraints applied energy efficient routing technique based on ant colony optimization used for disaster resilient location detection in mobile ad-hoc network", Journal of Ambient Intelligence and Humanized Computing, pp. 1-11, 2020.
[21] A. A. Junnarkar, Y. P. Singh, and V. S. Deshpande, "QMAA: QoS and Mobility Aware ACO Based Opportunistic Routing Protocol for MANET", In: Proc. of Computational Intelligence in Data Mining, Springer, Singapore, pp. 63-72, 2020.

[22] M. M. Mukhedkar and U. Kolekar, "E-TDGO: An encrypted trust-based dolphin glowworm optimization for secure routing in mobile ad hoc network", International Journal of Communication Systems, Vol. 33, No. 7, pp. 42-52, 2020.

[23] S. Mirjalili, S. M. Mirjalili, and A. Lewis, "Grey wolf optimizer", Advances in engineering software, Vol. 69, pp. 46-61, 2014.

[24] S. Mirjalili, S. Saremi, S. M. Mirjalili, and L. D. S. Coelho, "Multi-objective grey wolf optimizer: a novel algorithm for multi-criterion optimization", Expert Systems with Applications, Vol. 47, pp. 106-119, 2016. 